\title{
A contribuição de ensaios clínicos em psiquiatria: para além da ciência básica
}

\author{
The contribution of clinical trials in psychiatry: beyond \\ basic science
}

\begin{abstract}
Andrew Nierenberg
MD. Diretor médico, Programa de Clínica e Pesquisa em Transtornos do Humor Bipolar, Massachusetts General Hospital, Boston, MA, EUA. Diretor assistente, Programa de Clínica e Pesquisa da Depressão, Massachusetts General Hospital, Boston, MA, EUA. Professor assistente, Harvard Medical School, Boston, MA, EUA.
\end{abstract}

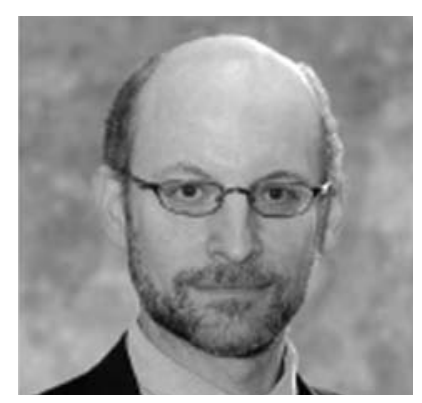

Parafraseando Dickens, é a melhor das épocas e a pior das épocas em termos de pesquisa em psiquiatria. É a melhor das épocas porque há uma explosão em ciência cerebral básica relevante para a psiquiatria: neurofisiologia, neurocircuitária, neurogênese, neuroproteção, neuroimagem funcional e estrutural, genômica, epidemiologia genética, interações geneambiente, farmacogenômica, proteômica e metabólica. E a pior das épocas devido à insuficiência de ensaios clínicos que avaliem novas intervenções psiquiátricas: poucos medicamentos novos ou psicoterapias inovadoras estão sendo desenvolvidos para depressão, transtorno bipolar, esquizofrenia ou transtornos de ansiedade. O financiamento a ensaios clínicos, sem ser feito por indústrias, sofreu redução considerável, com algumas exceções, nos últimos 5 anos.

Semelhante ao que havia ocorrido na medicina interna há uma geração, a psiquiatria está (adequadamente) preocupada com o entendimento da fisiopatologia para identificar defeitos essenciais que possam levar a novos alvos terapêuticos e a novos tratamentos. Entretanto, o número de ensaios clínicos psiquiátricos publicados na literatura que têm impacto sobre a prática sofreu uma redução. Além disso, o valor de ensaios clínicos tem sido questionado, motivado pelo ceticismo de que resultados negativos são removidos por empresas farmacêuticas e que essas mesmas empresas enfatizam benefícios mínimos ao mesmo tempo em que escondem os riscos de seus produtos. Os críticos vêm castigando as empresas farmacêuticas por perseguirem o lucro à custa da saúde dos pacientes ${ }^{1-3}$. Com todas essas questões em primeiro plano, qual seria então o valor da continuidade dos ensaios clínicos (se é que existe algum)?

Os ensaios clínicos geram evidências essenciais, embora imperfeitas, para que os clínicos tomem decisões informadas quanto ao cuidado de seus pacientes. Os ensaios clínicos são o método definitivo para testar se intervenções terapêuticas funcionam e oferecem dados importantes para guiar os clínicos na avaliação da relação risco/benefício. Esses ensaios testam as intervenções minimizando os vieses (por meio de randomização, controles e cegamento quando apropriado) - e perguntam: "a intervenção em estudo é melhor do que as alternativas (placebo ou controles ativos)?”. Tratamentos que parecem teoricamente atraentes devem passar pelo crivo de ensaios clínicos - simplesmente analise a história recente de intervenções promissoras que foram incapazes de demonstrar eficácia suficiente em ensaios clínicos (por exemplo, antagonistas do fator liberador da corticotrofina, estimulação magnética transcraniana, para citar alguns).

Os ensaios clínicos oferecem evidências para a “medicina baseada em evidências”. A expressão “medicina baseada em evidências” é, no entanto, peculiar. Qual é a alternativa? Utilizar medicina não baseada em evidências ${ }^{4}$ ? Os clínicos (devem) praticar a psiquiatria informados por ensaios clínicos (hard data), experiência clínica e raciocínio clínico (e, na verdade, através de relatos de casos de colegas) ${ }^{5-8}$. Mas os pesquisadores limitam a utilidade clínica dos resultados de ensaios clínicos relatando alterações médias em escalas de aferição de sintomas sem dados relevantes que identificariam aqueles pacientes que poderiam melhorar ou piorar durante um dado tratamento ${ }^{9}$. A generalização é outra questão ${ }^{10}$. Enquanto alguns pesquisadores descobriram que populações de pacientes clínicos e os de pesquisa são semelhantes ${ }^{11-13}$, outros descobriram que aqueles que buscam tratamento clínico diferem substancialmente daqueles que participam de pesquisas ${ }^{14}$. Visto que pode haver diferenças importantes entre populações clínicas e de pesquisa, os clínicos tendem a ser cuidadosos ao generalizar achados de pesquisas na prática diária e, ao integrarem sua experiência clínica com 
informações de ensaios clínicos randomizados (ECR), evitam transformar a medicina baseada em evidência em “medicina baseada em vieses"15*. Outras críticas aos ECR incluem: medidas de baixa sensibilidade, problemas com avaliadores de pesquisa e uso de projetos destinados a registro de medicamentos, ao invés de diretrizes para a prática clínica ${ }^{16,17}$. Contudo, os ECR, embora imperfeitos, servem como base essencial para informar a prática clínica. Os resultados não devem ser aplicados irrefletidamente, e a interpretação dos resultados deve considerar sua limitada validade externa.

A maioria dos ensaios clínicos psiquiátricos publicados são financiados e conduzidos por empresas farmacêuticas. As empresas conduzem os ensaios que julgam ser de seu melhor interesse e consistentes com seus modelos de negócio. As empresas farmacêuticas são a fonte da maior parte dos novos medicamentos e precisam conduzir os ensaios que finalmente servirão ao público e a seus acionistas - um modelo que tem tido enorme sucesso. Poucas intervenções, se é que há alguma, originam-se de fontes não-industriais. As empresas farmacêuticas tendem a evitar ensaios clínicos que possam prejudicar seu negócio - e as empresas muitas vezes evitam ensaios clínicos que diretamente comparam medicamentos e que possam apresentar o risco de tornar seu produto menos competitivo. O desafio é fazer com que as empresas realizem ensaios clínicos que, além de colocar medicamentos no mercado (ensaios de registro), também auxiliem na prática clínica diária, inclusive no desenvolvimento e teste de intervenções psicossociais. Fundações e governos desempenham importante papel no financiamento desses ensaios.

O Instituto Nacional de Saúde Mental dos EUA (NIMH) recentemente completou três grandes ensaios de efetividade, os quais foram planejados no final da década de 1990: CATIE, STEP-BD e STAR*D para comparar ou avaliar intervenções para esquizofrenia, transtorno bipolar e depressão, respectivamente. Cada estudo tem suas forças e limitações, apoiadores e detratores. Porém, a missão geral desses estudos foi conduzir ensaios clínicos que não seriam apoiados pela indústria e que trariam avanços no tratamento de doenças mentais graves. Esses tipos de estudos de efetividade oferecem uma forma diferente de informação do que os ensaios de registro, além de oferecer evidências adicionais para direcionar a prática clínica. Cada um desses grandes ensaios preparou uma infra-estrutura que se transformou em redes de ensaios clínicos para estudos futuros (redes de ensaios sobre esquizofrenia, transtorno bipolar e depressão).

Ensaios clínicos psiquiátricos desenhados com validação interna e externa que informem a prática

\footnotetext{
* Nota dos editores: Uma tradução livre em língua portuguesa que teria uma conotação irônica para o termo evidence-biased medicine poderia ser “medicina enviesada em evidências”.
}

clínica são dispendiosos, porém essenciais, se os clínicos querem ter acesso a dados para oferecer cuidados otimizados a seus pacientes. Precisaremos de financiamento contínuo e de mão-de-obra para conduzir ensaios clínicos que resultem em melhorias na saúde pública. O financiamento contínuo precisa de apoio público e, principalmente, de grupos de defesa. Precisamos de investimentos contínuos em programas de treinamento em pesquisa que formem pesquisadores clínicos para servir ao interesse público. Em resumo, os ensaios clínicos psiquiátricos são necessários para trazer avanços à prática clínica.

\section{Referências}

1. Avorn J. Powerful medicines: the benefits, risks, and costs of prescription drugs. New York: Vintage; 2005.

2. Angell M. The truth about the drug companies: how they deceive us and what to do about it. New York: Random House; 2005.

3. Kassirer JP. On the take: how medicine's complicity with big business can endanger your health. New York: Oxford University; 2005.

4. Isaacs D, Fitzgerald D. Seven alternatives to evidence based medicine. BMJ. 1999;319(7225):1618.

5. Devereaux PJ, Yusuf S. The evolution of the randomized controlled trial and its role in evidence-based decision making. J Intern Med. 2003;254(2):105-13.

6. Goldner EM, Bilsker D. Evidence-based psychiatry. Can J Psychiatry. 1995;40(2):97-101.

7. Sackett DL. Evidence-based medicine. Semin Perinatol. 1997;21(1):3-5.

8. Sackett DL, Rosenberg WM. On the need for evidence-based medicine. J Public Health Med. 1995;17(3):330-4.

9. Horwitz RI, Singer BH, Makuch RW, Viscoli CM. Can treatment that is helpful on average be harmful to some patients? A study of the conflicting information needs of clinical inquiry and drug regulation. J Clin Epidemiol. 1996;49(4):395-400.

10. Treatment Research in Mental Illness: Improving the Nation's Public Mental Health Care through NIMH Funded Interventions Research. Report of the National Advisory Mental Health Council's Workgroup on Clinical Trials. http://www.nimh.nih.gov/council/ interventions_research.cfm.p. 4.

11. Carroll KM, Nich C, McLellan AT, McKay JR, Rounsaville BJ. 'Research' versus 'real-world' patients: representativeness of participants in clinical trials of treatments for cocaine dependence. Drug Alcohol Depend. 1999;54(2):171-7.

12. Rapaport MH, Frevert T, Babior S, Zisook S, Judd LL. A comparison of demographic variables, symptom profiles, and measurements of functioning in symptomatic volunteers and an outpatient clinical population. Psychopharmacol Bull. 1995;31(1):111-4.

13. Stirman SW, DeRubeis RJ, Crits-Christoph P, Brody PE. Are samples in randomized controlled trials of psychotherapy representative of community outpatients? A new methodology and initial findings. J Consult Clin Psychol. 2003;71(6):963-72.

14. Braslow JT, Duan N, Starks SL, Polo A, Bromley E, Wells KB. Generalizability of studies on mental health treatment and outcomes, 1981 to 1996. Psychiatr Serv. 2005;56(10):1261-8.

15. Schelling FA. Clinical trials: deliberations on their essence and value. J Eval Clin Pract. 2004;10(2):291-6.

16. Fava G. The intellectual crisis of psychiatric research. Psychother Psychosom. 2006;75(4):202-8.

17. Wise TN. Curiosity and crisis. Psychother Psychosom. 2006;75(4):199-201.

\section{Correspondência:}

Andrew A. Nierenberg, Bipolar Program, Suite 580, 50 Staniford Street, 02140, Boston, MA, USA. E-mail: anierenberg@partners.org 